\title{
Petrographic and physical investigations on geomaterials for conservation of Las Plassas Castle (Marmilla, Sardinia, Italy) Stefano Columbua, Rita Teresa Melis ${ }^{\mathrm{a}}$, Giorgio Franco Murru', Giovanni Serrelic, Gabriella Uccheddu ${ }^{b}$ \\ ${ }^{a}$ Università degli Studi di Cagliari, Dipartimento di Scienze Chimiche e Geologiche, Cagliari, Italy, columbus@unica.it, ${ }^{\mathrm{b}}$ Centro di Documentazione e Comunicazione sul Castello di Marmilla e sulla civiltà rurale del Regno di Arborea tra il Basso Medioevo e l'Età Moderna (Las Plassas), Italy, 'Istituto di Storia dell'Europa mediterranea, C.N.R. di Cagliari, Italy.
}

\begin{abstract}
The Castle of Marmilla, located near the village of Las Plassas (central-Sardinia), represented a strategic military stronghold of great importance in the war between the kingdom of Arborea and the Aragonese Kingdom of Sardinia in the fourteenth century. Archaeological documents highlight the use, albeit partial, of some environments even in the modern age, during the times of feudal Sardinia (XV-XVI century), when the castle was an integral part of the possessions of the Barony of Las Plassas. It was used as a district prison until the nineteenth century. Built on top of a conical hill at 270 meters above sea level the castle has an irregular hexagonal shape, elongated along the north-west and south-east. Its foundations are excavated in a layer of Miocene sandstones and currently occupy the entire hilltop. What remains of the castle of Marmilla denotes a series of construction phases. A wide circle of walls delimiting the fortification of 550 square meters: inside there are the remains of a cistern and two towers, to the $\mathrm{N}$ and $\mathrm{S}$ sides, near the entrance. At present, the main tower and the walls, perimeter and internal, are preserved, albeit affected by a profound degradation, and they allow us to observe the construction techniques and choices made in the ongoing renovations, restorations and strengthening interventions. The structure is made of cantons sandstone cut with precision; at the base of the north tower was used ashlar stones. The cistern, located below ground level, is carved into the rock and covered with sandstone cantons; a second tank is located outside the masonry, near the northern side of the fortification.

By minero-petrographic and physical-mechanical analysis of geomaterials (i.e., stones, mortars) used in the castle construction, the alteration degree of the materials, in order to identify a correct restoration for conservation purposes, has been defined.
\end{abstract}

Keywords: Minero-petrographic analysis, Physical properties, Alteration, Sandstone

\section{Introduzione storica}

Il Castrum Mamilla o Marmilla (Fig. 1), detto dal Fara Castrum Platearum e oggi noto come Castello di Las Plassas, è impiantato sulla sommità di una collina di forma conica $(274 \mathrm{~m}$ s.1.), situata nelle vicinanze dell'abitato di Las Plassas nella regione storico-geografica di Marmilla che prese il nome dal castello
(Murru, 1988-89; Serreli, 1999-2000; Serreli, 2000, 1; Serreli, 2002).

Non si conosce la data esatta di erezione del castello che, unitamente a quelli di Arcuentu e di Monreale, costituiva la linea fortificata tra il Regno di Arborèa da quello di Càlari; 
verosimilmente fu costruito subito dopo la nascita dello Stato verso il X secolo. Ma la sua importanza strategica confermata dagli avvenimenti storici dei secoli medievali e della prima età moderna, è da attribuire oltre che al presidio del confine meridionale del Regno arborense (Serreli, 2006; Serreli, 2010), anche al controllo della via di comunicazione lungo il Calarita flumen (Flumini Mannu) fra le pianure del Campidano e le montagne del Sarcidano e, soprattutto, della fertile pianura omonima da sempre ricca produttrice di cereali e leguminose (Murru, 1988-89). La documentazione storica ci conferma l'importanza strategica ed economica di questa regione, non a caso presidiata prima dalla reggia nuragica di Barumini e poi dal castello medievale; le recenti indagini geoarcheologiche, condotte dai proff. Rita Teresa Melis (Università di Cagliari), Charles French (Università di Cambridge) e Federica Sulas (ISEM CNR), confermano un intenso utilizzo agricolo, senza soluzioni di continuità, della fertile pianura controllata dal castello.

Il Castrum compare per la prima volta nella documentazione storica tra il 1168 e il 1172, quando il re Barisone $I$ di Arborea s'impegnava a cedere in pegno i castelli di Arcuentu e di Marmilla alla Repubblica di Genova per il debito contratto in seguito agli accordi per farsi incoronare rex Sardiniae da Federico Barbarossa (Serreli, 2000; Serreli, 2003). La fortezza rimase in mani liguri sino al 1195 , anno in cui il re calaritano GuglielmoSalusio IV invase il territorio arborense. Terminato il conflitto, il 30 ottobre 1206 le frontiere fra i due Stati furono ridefinite ma il castello rimase in mani arborensi; infatti, quando nel 1228 il sovrano di Arborèa Pietro II fece un'ampia donazione ai monaci Benedettini, fra $\mathrm{i}$ testimoni viene nominato Guantini de Zori castellanu de Marmilla, il quale rivestì questa carica almeno fino al 1237 (Murru, 1992; Serreli, 2000, 2).

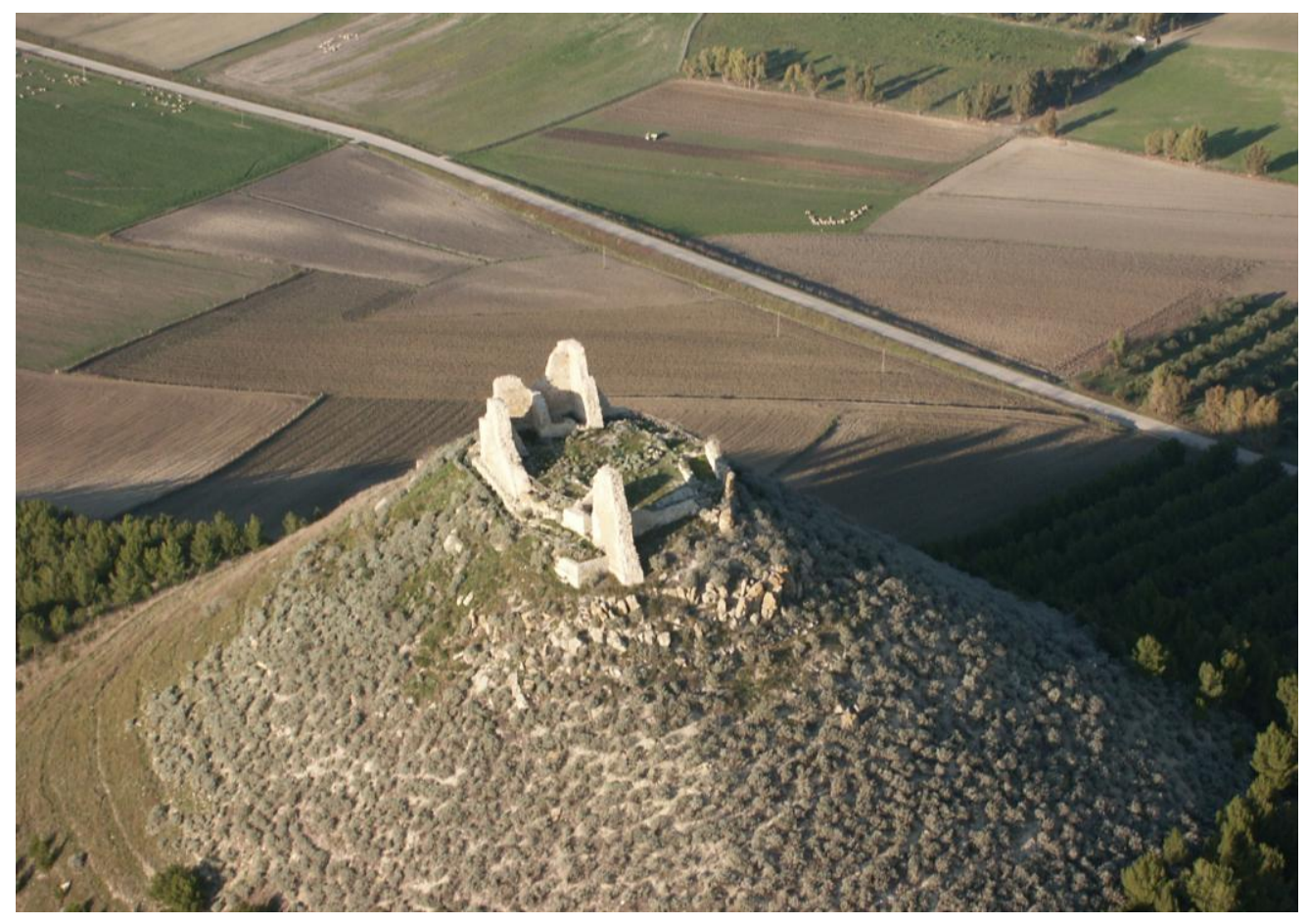

Fig. 1- Vista aerea del Castello Marmilla (noto anche col nome di Castello di Las Plassas) 
A esperte maestranze pisane sono attribuiti i consistenti restauri che, tra la fine del XIII e gli inizi del XIV secolo, interessarono le strutture del castello le quali avevano subito danni a causa delle frane della collina argillosa, ma forse anche a causa di una campagna militare con cui Nino Visconti nel 1297 cercò di recuperare il Regno di Gallura. Questo restauro è accostabile per tecnica costruttiva alla torre di S. Cristoforo di Oristano, del 1290. Intorno al 1304, per un breve periodo, il castello passò sotto il controllo dei Pisani. Tornò poi sotto la potestà del Regno di Arborèa, visto il trattato di alleanza del 1323 fra i due re Ugone II de Bas-Serra e Giacomo II della Corona d'Aragona è enumerato fra i possessi arborensi (Murru, 1988-89; Serreli, 2003).

Nel 1331 Ugone II nominò il suo secondogenito Mariano de Bas Serra (futuro Mariano IV) signore di Marmilla e conte di Goceano; con questa carica il principe, futuro re, fin dal 1340 iniziò una serie di azioni di guerriglia contro il Regno di 'Sardegna e Corsica' aggregato alla Corona d'Aragona, mettendo in discussione l'alleanza siglata da suo padre; in questi anni il castellano di Marmilla è tale Gonnarius de Sogiu.

L'alleanza fra il Regno di Arborèa e il Regno di Sardegna presto si ruppe definitivamente, e agli inizi di settembre del 1353 le mudas guerriere di Mariano IV, comandate fra gli altri da Cino de Zori, già castellano di Marmilla, invasero il calaritano. Nelle trattative di pace che seguirono a Sanluri l'11 luglio 1355, Pietro il Cerimonioso chiese che a capo dei castelli di Montiverro e Marmilla fossero posti due castellani di nazionalità catalana o aragonese prestanti a lui giuramento di vassallaggio; ma Mariano IV non ottemperò mai a quanto pattuito (Serreli, 2000, 2).

Ritroviamo ancora il castello di Las Plassas nel 1388, nel documento riguardante il trattato di pace stipulato tra Eleonora d'Arborea e il nuovo re del Regno di Sardegna Giovanni I il Cacciatore (castellano era allora Nicolao Longu). Alcuni mesi dopo la battaglia di Sanluri del 1409, dopo aver resistito alcuni mesi, il castello entrò a far parte del Regno di
Sardegna. I governanti iberici provvidero subito a rifornirlo e dotarlo di quanto necessario alla difesa e, nell'ambito di una riorganizzazione dell'apparato difensivo, ai castelli di Monreale e Marmilla, ritenuti di primaria importanza per il controllo del meridione dell'isola, veniva assegnata la maggior parte delle risorse stanziate.

Nel 1412 il castello venne affidato al fedele castellano Gondissalvo de Carmona. Già il 9 gennaio 1412 il governatore del Capo di Cagliari e Gallura, Berengario Carròs conte di Quirra, ordinava all'amministratore Pietro Bernes di rifornire «...los castells de Munt Reyal, de Marmilla e de Sentllurj...» di grano, vino, aceto, olio, legumi e altre vettovaglie e di quant'altro avessero avuto bisogno; lo stesso governatore, 1'11 febbraio 1413, reiterò l'urgente richiesta, specificando che i castelli in questione avevano bisogno di quattro casse di chiavistelli, una balestra, sei dozzine di corde di balestra, due giare d'olio, tre starelli di legumi, milleduecento mazzi di aglio e acqua per cuocere alimenti (Serreli, 2003, 1; Serreli, 2010). In quegli anni Berengario Carròs teneva occupata militarmente la Marmilla con il pretesto di difenderla dal sovrano di Arborèa, Guglielmo di NarbonaBas. In realtà, il Carròs aspirava ad averla in concessione feudale, tanto che nel Llibre de Registre dels Privileges del Marquesat de Quirra, del 1504, si legge che il «castro de Les Places» e la «villa de Places» appartenevano sin dal 1414 a Berengario (Serreli, 2000, 2).

Intanto la Marmilla era richiesta in feudo anche da Luigi de Pontos, governatore del Capo di Cagliari e Gallura dal 1417 e viceré nel 1418; ma inutilmente. Nel 1415-16 il nuovo sovrano Alfonso il Magnanimo pensò di infeudare il castello di Marmilla a Garçia de Ferrera, cavaliere barcellonese giunto nell'isola al seguito di Pietro Torrelles; ma, con lettere del 1416 e 1417, i consiglieri di Cagliari si opposero con decisione, affermando che tali territori appartenevano alla loro Città in virtù di privilegi concessi da Pietro il Cerimonioso durante il Parlamento del 1355, confermati nel 1366, per i quali non si potevano concedere o alienare ad alcuno terre 
del Patrimonio regio ricadenti nella giurisdizione del Capo di Cagliari-Gallura (in sostanza, gli abitanti della capitale del Regno non volevano privarsi delle alte risorse che la Marmilla garantiva loro). Così, Garçia de Ferrera morì due anni dopo, senza essere entrato in possesso del suo bene.

Nel 1417, l'ufficio di castellano venne affidato a Alfonso Periç di Valladolid, un militare che rifiutava la giurisdizione del governatore, accendendo una lunga vertenza superata solo vent'anni dopo, quando il 14 giugno 1437 il re Alfonso il Magnanimo emanò un documento di conferma in favore del marchese di Oristano, Antonio Cubello, circa i feudi, i privilegi e le concessioni fatte al padre Leonardo nel 1410 (oltre al marchesato di Oristano e al contado di Gocèano, erano confermate al Cubello e alla sua discendenza «castrum Montis Regalis et castrum Marmillae, nec minus villas, curatorìas, terras, tenimenta et pertinentias civitatum et castrorum praedictorum»). In realtà il castello restò sempre nelle pertinenze dei feudatari che controllavano il feudo di Las Plassas (i de Moncada dal 1421; i Besalù dal 1458; gli Zapata dal 1541 all'abolizione del feudalesimo nel 1839, Serreli, 2000, 2).

In questi secoli venne meno l'importanza strategica della fortezza che rimase parzialmente attiva come carcere della Baronìa di Las Plassas, fino agli inizi del XIX secolo.

\section{Gli studi e l'intervento archeologico}

La struttura architettonica del Castello di Marmilla (Fig. 2) domina la piana omonima e ha sempre suscitato l'interesse degli studiosi.

Il castello fu oggetto di analisi delle strutture murarie fuori terra alla fine degli anni ' 80 del $\mathrm{XX}$ sec. Il prezioso studio portò all'individuazione di più fasi costruttive e importanti restauri che interessarono sia $i$ perimetrali che la torre principale a Nord del mastio, individuando una cronologia relativa compresa tra il XIII e il XVI sec. (Murru, 1988-89).

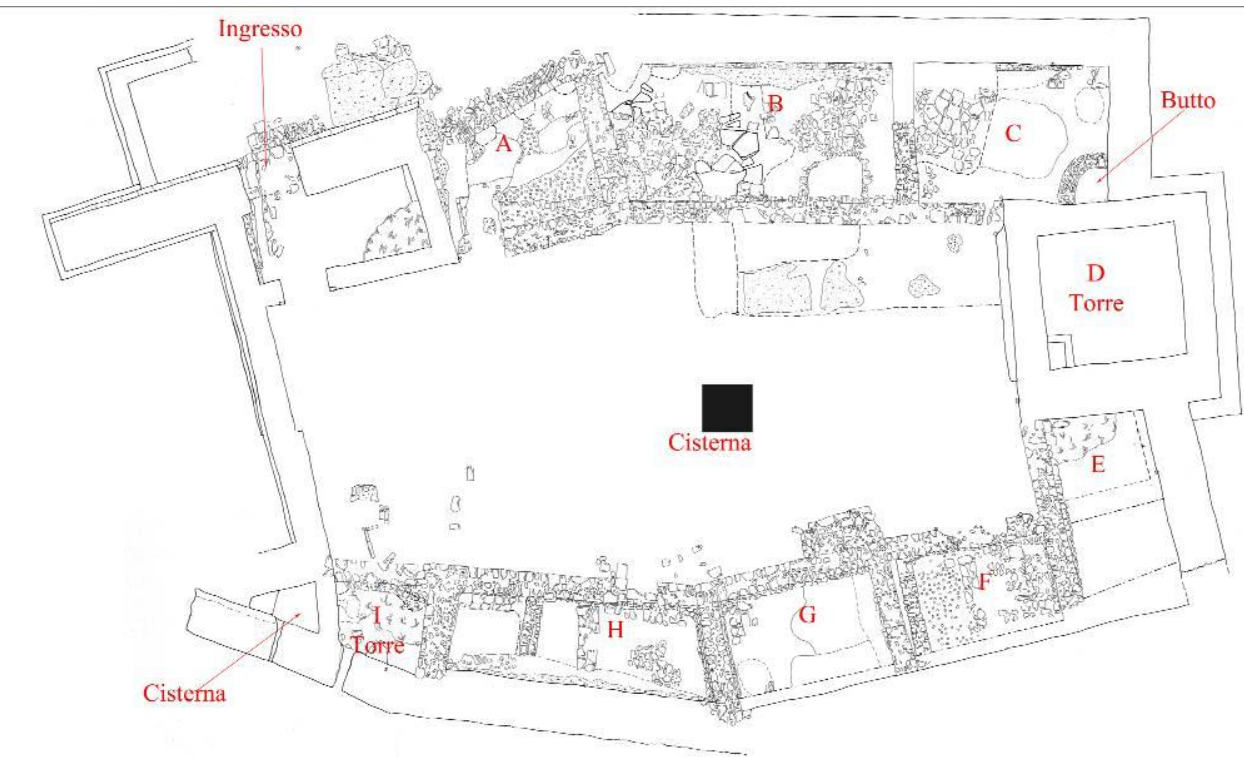

Fig. 2- Planimetria del Castello Marmilla con suddivisione degli originari ambienti

A questo lavoro di analisi strutturale, nel 2000 seguirono i lavori di restauro e ripristino dei perimetrali Nord, Ovest e Sud, nonché la rimozione dei crolli su tutta l'area che portò 
alla definizione planimetrica del castello individuando così un'area di circa $550 \mathrm{mq}$ e una planimetria di forma esagonale allungata. Il cassero è caratterizzato da una corte centrale o piazza d'armi, dov'è ubicata una cisterna ipogeica di notevoli dimensioni con pianta quadrangolare, dotata di una copertura a botte.

Sono state individuate tre torri (Fig. 2): quella denominata $\mathrm{D}$ è posizionata a Nord; la seconda è ricavata dall'andamento semicircolare dell'incontro del perimetrale Est e quello Sud (ambiente I), mentre la terza è adiacente all'ingresso. I perimetrali non presentano nessun affaccio verso l'esterno, le aperture degli ambienti si aprono tutte sulla corte centrale o piazza d'armi. Nel 2007, considerato l'interesse del monumento, l'amministrazione comunale di Las Plassas, in accordo con la Soprintendenza archeologica, avviò la prima campagna di scavo archeologico all'interno del castello. Dati i tempi ristretti, non tutti gli ambienti furono indagati esaustivamente. Ogni ambiente è identificato con una lettera e il percorso avviene in senso orario (Fig. 2).

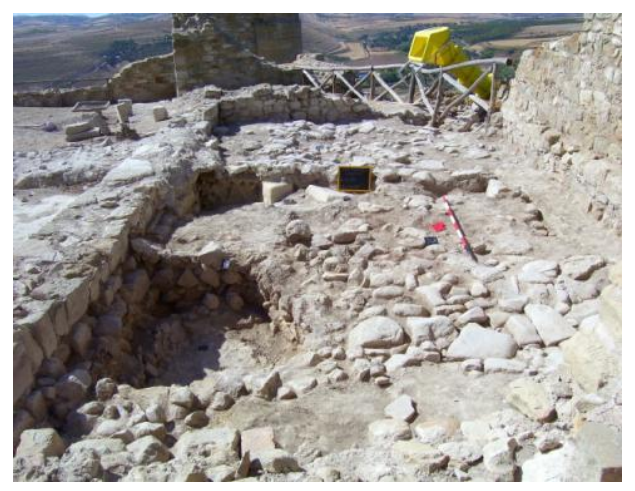

Fig. 3- Vista degli scavi degli ambienti A (vano in lontananza) e B (in primo piano).

Il lavoro ha permesso di individuare la funzione di alcuni ambienti e i relativi piani di frequentazione. L'ambiente denominato $\mathrm{B}$ (Fig. 3), probabilmente tra il XIII e il XIV secolo è stato adibito a "fucina"; presenta una struttura interrata di forma ellittica, con fondo leggermente concavo. La struttura presenta evidenti tracce di bruciato dovute a un ripetuto contatto con le alte temperature. Il fondo è costituito da strati di battuto argilloso ben compattati, alternati a strati di carboni e frammenti ossei in parte combusti. Sul fondo non sono state trovate tracce di metallo fuso o scorie di lavorazione dei metalli ma la pavimentazione in acciottolato dell'ambiente presenta sulla superficie chiazze di materiale ferroso e questo ha fatto ipotizzare che la struttura sia servita in qualche processo di lavorazione dei metalli.

Di particolare interesse archeologico è il vano adiacente, denominato $\mathrm{C}$, dove nell'angolo di $\mathrm{NE}$ è stato individuato un immondezzaio medievale, contenuto all'interno di una struttura muraria con andamento semicircolare. Il "butto" ha restituito resti di pasto (ossa, squame di pesce e gusci d'uova) e un notevole quantitativo di ceramiche smaltate acrome databili tra il XIV e inizio del XV sec. (Carrada, 2003).

Sull'altro lato della corte ad Est è stata individuata la cucina (vano G). Il piano di calpestio è costituito da terra argillosa di colore giallo, caratterizzato da notevoli tracce di focolari (ceneri e carboni) e ha restituito piccole quantità di ceramiche grezze da fuoco. Risulta di notevole interesse archeologico l'ambiente adiacente di Sud Est, denominato $\mathrm{H}$, in parte ipogeico e ha subito nel tempo numerosi vicissitudini costruttive e funzionali. Data l'esposizione a SE, è ben ventilato, caratteristica che fa ipotizzare un utilizzo nel medioevo come magazzino per lo stoccaggio di derrate alimentari.

\section{Inquadramento geologico-geomorfologico dell'area}

Il quadro geologico dell'area (Fig. 4), dove sorge il Castello Marmilla, è caratterizzata da un substrato rappresentato dalla successione sedimentaria miocenica della Formazione della Marmilla (Cherchi, 1985).

Questa è costituita da un'alternanza di siltiti e arenarie, marne siltitiche con abbondante contenuto in foraminiferi e altri organismi planctonici. Talvolta si rinvengono bancate di arenarie medio-grossolane e fini. Nei livelli arenacei sono presenti elementi vulcanogenici, generalmente con minerali quali biotiti e feldspati. 

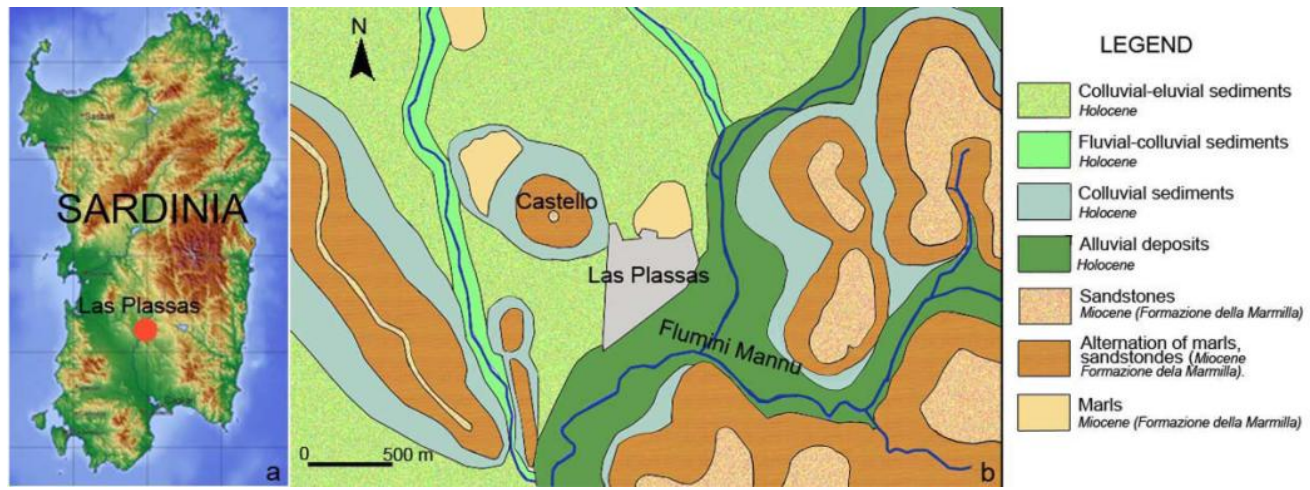

Fig. 4- a) Localizzazione del Castello Marmilla nella Sardegna centro meridionale in prossimità del paese di Las Plassas; b) schema geologico dell'area oggetto di studio con relativa legenda delle principali litologie presenti

La monotonia della successione è interrotta dalla variazione degli spessori dei livelli arenacei e marnosi, oppure per l'abbondanza di minerali di origine vulcanica. Sono inoltre frequenti banchi di arenarie grossolane a forte componente vulcano-clastica e scarsa cementazione carbonatica (tufiti arenacee) associate a livelli di tufi cineritici contenenti localmente pomici alterate. Depositi alluvionali sono presenti lungo la piana alluvionale dei principali corsi d'acqua e lungo i letti attuali.

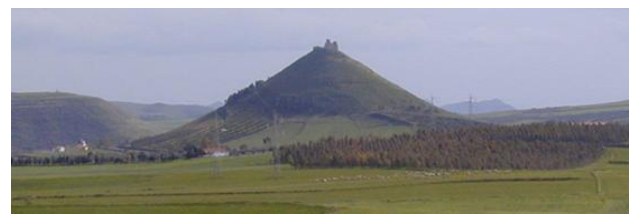

Fig. 5- Collina di Las Plassas, su cui si erge il Castello Marmilla, circondata da rilievi a cuestas.

Il contesto geomorfologico dell'area è caratterizzato da colline isolate a forma di cuestas (Fig. 5) con versanti a gradoni dove affiorano le testate degli strati dei litotipi della successione sedimentaria miocenica.

\section{Caratterizzazione dei materiali e metodologie di studio}

\subsection{Obiettivi}

Il presente lavoro verte sullo studio dei geomateriali utilizzate per la costruzione del castello di Marmilla.
Tra gli scopi della ricerca, l'interesse volge allo studio dei processi di alterazione sia di tipo fisico che chimico che interessano alcune litologie facilmente attaccabili dal degrado (e.g., vulcaniti pomiceo-cineritiche; Columbu et al., 2014; Coroneo \& Columbu, 2010; Macciotta et al., 2001), e le malte utilizzate nei vari originari ambienti del castello. Al momento, le indagini (attualmente in corso) si rivolgono in modo specifico alle malte di allettamento dei conci regolari ed informi e degli intonaci (Fig. 6) delle murature degli ambienti I (torre) e H (probabile magazzino per le derrate alimentari; Fig. 2). Questo vano, in parte ipogeico, risulta essere di particolare interesse in quanto ha restituito una cronologia abbastanza precisa degli elevati, compresa tra il XII e il XVI secolo. E' un ambiente che ha subito nel tempo numerosi interventi di restauro, tuttora ben leggibili grazie alle differenti tessiture murarie impiegate, diventa conseguentemente un ottimo caso-studio per verificare le variazioni composizionali nel tempo delle malte e le diverse tecnologie costruttive impiegate. Inoltre, l'analisi anche soltanto dei rapporti di miscelazione tra aggregato e legante, così come di alcuni componenti dell'aggregato possono fornire informazioni utili sui cambi (anche in tempi relativamente brevi) delle maestranze che ha lavorato sulla edificazione del castello. 


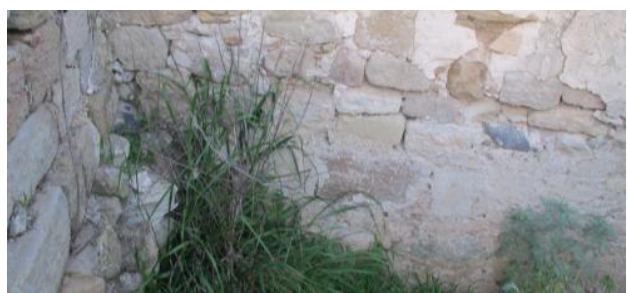

Fig. 6- Tessitura muraria dove sono visibili le malte di allettamento, gli intonaci e le litologie.

\subsection{Materiali e metodi analitici}

Delle diverse litologie e delle malte presenti nelle murature della struttura (Fig. 6) sono stati prelevati campioni di dimensioni centimetriche (in alcuni casi ancora integri), su cui eseguire le analisi minero-petrografiche $\mathrm{e}$ fisiche. Il campionamento delle malte è stato impostato secondo criteri di vario genere: quota dal piano inferiore (calpestabile) del vano $\mathrm{H}$ (Fig. 2), funzione diversa nell'ambito della struttura (malta di allettamento, d'intonaco, etc.), grado di alterazione, varietà composizionale determinata attraverso analisi macroscopica, profondità orizzontale (corrispondente ai diversi strati di finitura nel caso degli intonaci). Questo metodo di campionamento consente di evidenziare le probabili variazioni composizionali (tipo di aggregato, rapporto legante/aggregato, etc.) delle malte in relazione alle diverse fasi di costruzione e/o a cambiamenti nel tempo delle maestranze o delle tecnologie costruttive (Columbu et al., 2015). Di ogni campione in laboratorio sono stati realizzati:

- provini pseudo-prismatici (con dimensione media $<20 \mathrm{~mm}$ ) su cui determinare le proprietà fisiche (densità reale ed apparente, porosità, assorbimento d'acqua per immersione, indice di saturazione, resistenza meccanica, etc);

- sezioni sottili con spessore di circa 30 micron per la determinazione mediante microscopio polarizzatore delle caratteristiche petrografiche dell'aggregato delle malte e delle varie litologie (gli aspetti micro-strutturali e tessiturali, la composizione mineralogica);

- campioni macinati per l'eventuali analisi chimiche mediante spettrometro per fluorescenza a raggi $\mathrm{X}(\mathrm{XRF})$ e le analisi diffrattometriche (XRD).

Per le prove fisiche, sono stati utilizzati un picnometro ad elio (modello Ultrapycnometer 1000, Quantachrome Instruments), una bilancia analitica idrostatica per le masse sature e secche e un Point Load Tester.

\section{Discussione dei risultati e conclusioni}

L'indagine archeologica, seppure non completamente esaustiva degli ambienti del Castello Marmilla, ha consentito la comparazione dei dati scientifici emersi dallo studio delle strutture murarie e lo studio preliminare dei materiali archeologici rinvenuti in fase di scavo. L'analisi dei dati ha evidenziato un utilizzo continuativo del maniero in un periodo tra il XII e il XVI secolo, del tutto compatibile con quanto affermano le fonti. Gli oggetti più significativi fanno parte di un suggestivo percorso museale multimediale, all'interno del MudA di Las Plassas, dove il visitatore può immergersi in aspetti della vita nel medioevo arborense e nel castello di Marmilla.

L'analisi geomorfologica del territorio intorno al Castello Marmilla ha evidenziato un'area caratterizzata da colline isolate a forma di cuestas con versanti a gradoni dove affiorano le testate degli strati dei litotipi della successione sedimentaria miocenica affiorante nella zona. Particolarmente caratteristica è la forma perfettamente conica dove sorge il castello, testimone dei processi di erosione che hanno interessato tutta l'area durante il PlioPleistocene. I processi di erosione dei versanti della collina sono legati al dilavamento delle acque superficiali e ai processi gravitativi che hanno causato piccole frane di scivolamento. Questi fenomeni resi più intensi dall'uso agricolo, hanno causato una maggiore erosione dell'alto e medio versante e depositi colluviali nel basso versante. Questi processi sono anche la causa dell'arretramento per frane del banco di arenarie su cui poggia il castello determinando conseguentemente un dissesto strutturale (già a partire dall'epoca storica) con arretramento della struttura. Lo studio preliminare delle malte di allettamento e d'intonaco delle murature degli 
ambienti $\mathrm{H}$ ed I del castello, rispettivamente riferiti al probabile magazzino di derrate alimentari e ad una delle tre torri, ha evidenziato la presenza di malte di allettamento in alcuni casi fortemente alterate, soprattutto quelle posizionate nelle parti basali della struttura, con un legante di scarsa consistenza e conseguenti basse resistenze meccaniche. Nella realizzazione degli intonaci sono stati utilizzati due diversi impasti con differenti funzioni: uno per lo strato di arriccio (in alcuni casi anche di rinzaffo) ed un impasto più omogeneo composizionalmente per lo strato di finitura.

\section{References}

Carrada F. (2003). Studio preliminare dei reperti dal castello di Marmilla (Las Plassas, Cagliari), in Roccas. Aspetti del sistema di fortificazione in Sardegna (Atti degli Incontri sui Castelli in Sardegna (2002) dell'Arxiu de Tradicions), a cura di Sara Chirra, Oristano, pp. 77-96.

Columbu S., Gioncada, A., Lezzerini, M., Marchi, M. (2014). Hydric dilatation of ignimbritic stones used in the church of Santa Maria di Otti (Oschiri, northern Sardinia, Italy). Ital. J. Geosci. (Boll. Soc. Geol. It.), Vol. 133, 1, pp. 149-160.

Columbu S., Sitzia F., Verdiani G. (2015). Contribution of petrophysical analysis and 3D digital survey in the archaeometric investigations of the Emperor Hadrian's Baths (Tivoli, Italy). Rendiconti Lincei, Springer. In press

Coroneo R., Columbu S. (2010). Sant'Antioco di Bisarcio (Ozieri): la cattedrale romanica e i materiali costruttivi. Archeoarte, vol. 1, p. 145-173, ISSN: 2039-4543, doi: DOI: 10.4429/j.arart.2010.01.10

Coroneo R. (1993). Architettura romanica dalla metà del Mille al primo '300, collana "Storia dell'arte in Sardegna", Nuoro, Ilisso, sch. P. 174.

Macciotta G., Bertorino G., Caredda A., Columbu S., Coroneo R., Franceschelli M., Marchi M., Rescic S. (2001). The S. Antioco of Bisarcio Basilica (NE Sardinia, Italy): water-rock interaction in ignimbrite monument decay. In: WRI-10, Cidu Ed., Swets \& Zeitlinger, Lisse, vol.1, p. 415-418.

Murru G.F. (1988-89). Il castello di Marmilla o Las Plassas. Cenni storici e architettonici, in "Studi Sardi”, XXIX, pp. 393-424.

Murru G.F. (1992). Un mucchio di rovine ricche di storia, in F. Fois Castelli della Sardegna Medioevale, a cura di B. Fois, Cinisello Balsamo, Amilcare Pizzi, p. 144.

Serreli G. (1999-2000). Las Plassas. Un'insediamento rurale tra l'Età Antica ed il Medioevo, in «Almanacco Gallurese» n 7, pp. 60-63.

Serreli G. (2000, 1). Il periodo giudicale, in Dentro la Marmilla. Amb. Storia Cult., Dolianova, pp. 3543.

Serreli G. $(2000,2)$. Las Plassas. Le origini e la storia, Serramanna.

Serreli G. (2002). Il rinvenimento di un 'iscrizione dedicatoria dei pagani Uneritani a Las Plassas, XIV volume de L'Africa Romana (Atti Convegno Internazionale, Sassari 7-10 dicembre 2000), a cura di M. Khanoussi, P. Ruggeri e C. Vismara, Carocci Editore, Roma, III vol., pp. 1787-1793.

Serreli G. (2003, 1). Rifornimenti ai castelli di Sanluri, Monreale e Marmilla o Las Las Plassas tra il 1412 e il 1413, in Aragonensia. Quaderno di studi sardo-catalani, Dolianova, pp. 11-15.

Serreli G. (2003, 2). Il castello di Marmilla a Las Plassas in Roccas. Aspetti del sistema di fortificazione in Sardegna, a cura di Sara Chirra, Oristano, pp. 71-76.

Serreli G. (2004). Las Plassas nella Guida ai 20 Comuni - Sa Corona Arrùbia, Bolzano, pp. 74-83.

Serreli G. (2006). Tutti $i$ castelli dei quattro Regni, in «Darwin. Quaderni» n. 1 - Archeologia in Sardegna, Roma, pp. 104-109.

Serreli G. (2010). La frontiera meridionale del Regno giudicale d'Arborea: un'area strategica di fondamentale importanza per la storia medievale sarda, in "RiMe. Rivista dell'Istituto di Storia dell'Europa Mediterranea" n. 4. 